\title{
The application of quality improvement methodologies in surgery
}

\author{
Stephanie Fong \\ Faculty Reviewer: Sayra Cristancho, PhD (Centre for Education Research \& Innovation)
}

\section{ABSTRACT}

Quality improvement (QI) practices were originally developed in the manufacturing industry to reduce unnecessary steps in a process, minimize error, and provide maximum benefit to the consumer. QI is defined as a formal approach to the analysis of performance and systemic efforts to improve it. QI methodologies have been adopted by industries outside manufacturing, including healthcare. In the healthcare environment, performance consists of many factors including patient safety, clinical results, and system efficiency. Given the publicly funded, limited resource environment in which the Canadian healthcare system operates, the practice of delivering safe, quality healthcare in an efficient and cost-effective manner is an important factor in promoting the economic viability and sustainability of the system. Surgical practice has been identified as an area in which QI methodologies can be applied, given its resource intensive nature and highly regulated environment. Current research supports the use of QI in surgery, with interventions showing improvements in non-operative time, on-time starts, and operating room patient volume. Limitations to the application of QI include the heterogeneity of interventions and variability in terms of procedures and patient factors. Further high-quality studies are required to support evidence-based applications of $Q I$ in the surgical setting as well as the greater healthcare environment.

\section{INTRODUCTION}

In Canada's publicly funded single-payer healthcare system, the challenge of allocating limited healthcare dollars across a growing population with increasingly complex medical needs has healthcare leaders working to identify ways to improve system efficiency without compromising care. Increasing healthcare demands along with rising treatment and technology costs have led to growing funding concerns. ${ }^{1}$ The Canadian Institute for Health Information (CIHI) estimates health expenditures to reach $\$ 228$ billion or $\$ 6,299$ per person, representing $11 \%$ of Canada's gross domestic product (GDP) in 2016. Hospitals make up the largest proportion of health care spending in Canada at $29.5 \%{ }^{2}$

Funding challenges in combination with the need to provide high quality patient care in a fixed resource environment has led to the increasing application of quality improvement (QI) methodologies from industry. ${ }^{3}$ In the healthcare setting, surgical practice is an area that presents opportunities for improved efficiency as it requires a regimented environment and is inherently resource in- tensive. Small, medium, and large-scale QI initiatives have been introduced in the surgical setting with the goals of reducing wait times, improving operating room (OR) efficiency, decreasing operative complications, limiting unnecessary costs, and minimizing hospitalization duration. The largest portion of surgical dollars is allocated to the $\mathrm{OR}^{4}$, making it a high priority for $\mathrm{QI}$ initiatives. The purpose of this article is to provide a succinct review of QI methodologies and their application in surgery.

\section{QUALITY IMPROVEMENT METHODOLOGIES}

There are several QI methodologies adopted by healthcare from the manufacturing industry, with select examples listed below:

Continuous quality improvement (CQI) developed from statistical process control, a method of quality control using statistical methods. This management philosophy incorporates quality into processes at the outset, rather than addressing issues as they arise. CQI is used to reduce waste, increase efficiency, and boost internal and external satisfaction. Quality improvement is derived from continuously evaluating performance. ${ }^{1}$

Total quality management (TQM) is a management approach to achieve consumer expectations though continuous improvement in the quality of products and processes. In TQM, everyone involved in the production or use of a product or service is responsible for quality and participates in improving processes, products and services, and workplace culture. TQM practices include consumer feedback, committed leadership, employee involvement, process management, and strategic planning. ${ }^{1}$

Plan-Do-Check-Act (PDCA) or Plan-Do-Study-Act (PDSA) is an iterative process involving a four-step system to monitor the effect of a change over time. The initial "planning" step includes establishing the objectives and processes to deliver results aligned with the desired output. The second step, "do", involves smallscale implementation of the new process. The third step, "check" or "study", requires observing differences between the desired and observed outcome. The final step, "act", involves analyzing the difference between expected and observed results and determining the cause of the disconnect. The process is then repeated. ${ }^{1}$

Lean methods ("lean") originated in the Japanese automotive manufacturing sector in 1990 with the Toyota production system. ${ }^{4}$ Lean aims to eliminate waste in a system by removing unnecessary steps in a process pathway while preserving steps that maximize value to the consumer. ${ }^{3,5,6}$ Other lean principles include reducing the time and resources required to generate results for the consumer and improving the system's ability to respond to the changing 
needs of the consumer. ${ }^{7}$ Value stream mapping in lean management methods analyze the flow of information and materials required to produce a product or service. ${ }^{7}$

Six sigma was developed in 1986 by the Motorola Corporation and aims to eliminate defects in a system by reducing process variation through identifying and correcting the causes of errors. The methodology requires constant data collection and statistical analysis. The ultimate goal is to operate at a six sigma level, 3.4 defects per million opportunities (DPMO). ${ }^{1,3,8}$

Lean six sigma was created in the early 2000s and combines the waste elimination principle of lean management methods with the defect reduction focus of six sigma. The two methodologies are combined in the DMAIC (Define, Measure, Analyse, Improve, Control) toolkit., ${ }^{1,3}$

\section{QUALITY IMPROVEMENT IN SURGERY}

There are a variety of QI interventions that have been applied to the surgical setting. Depending on the scale of the initiative, the degree of human capital and hospital resource requirements vary. Small-scale interventions can be employed relatively quickly and require limited human capital. ${ }^{9}$ An example of a small-scale project is standardization of operating instruments, which has been shown to reduce set up time, counting time, turnover time, and operative costs. Medium-scale interventions require buy-in from a larger group of people, one example being an entire OR floor or group of practitioners. The concept of teaming, where surgeons work with fixed teams on similar procedures, can reduce OR time. Cases run more efficiently as teams have an increased familiarity with instruments and surgeon preferences, as well as an increased ability to anticipate needs. An example of a large-scale intervention was studied at Massachusetts General hospital where an "OR of the Future (ORF)" was created to investigate physical space redesign on efficiency. In this study, induction and recovery rooms were adjoined to improve patient flow and patient beds doubled as gurneys, reducing patient transfers and improving throughput. The ultimate result of the ORF was increased capacity to perform two additional procedures daily. ${ }^{9}$ Lean processes and six sigma strategies, which aim to reduce waste and eliminate defects in a system, respectively, are two of the most recognized QI methodologies in the manufacturing industry and have been applied to large healthcare initiatives with positive results.

In 2013, the Veteran's Health Administration (VHA) designed several pilot projects in collaboration with lean experts with the goal of decreasing wait times, improving access to care, and increasing scheduling efficiency though the implementation of lean processes. ${ }^{7}$ The Richard L. Roudebush Veterans Affairs Medical Center is a tertiary centre in Indianapolis that focused specifically on reducing surgical wait times within the general surgery service. Initial steps involved the meeting of a multidisciplinary group including surgeons, nurses, OR staff, administrators, and lean experts to identify and map out a value stream that included current inefficiencies, delays, and communication breakdowns. The team then created an idealized value stream and developed strategies to achieve this future state with the goal of improving access and reducing wait times. Specific areas targeted included standardizing work across surgical subspecialties, restructuring consultation methods, and improving interdepartmental communications. Results of the study demonstrated increases in patient volume both in general surgery clinic and in the OR.

Lean and six sigma strategies were also used during a 2008 initiative undertaken at the Mayo Clinic to enhance operating room efficiency. ${ }^{8}$ A global assessment of patient flow from the initial surgical consult to post-operative recovery was conducted. The initial step in this process was to create a value stream map of patient flow that detailed location, personnel involved, information technology requirements, and bottlenecks. Objectives were then identified by a multidisciplinary team and included reducing non-operative OR time, decreasing over and underuse of OR resources, reducing the redundant collection of patient information, and enhancing employee engagement. Strategies such as parallel processing to decrease non-operative OR time and working with information technology programmers to develop standardized data collection and terminology across electronic applications were employed. One of the largest barriers to the success of QI initiatives was employee engagement. This barrier was addressed by the creation of a multidisciplinary communication council that acted to resolve differences between various stakeholder groups and establish clear expectations for specific roles. Results from this project included improvements in non-operative time, improvements in on-time starts, and reductions in the number of cases past scheduled OR time. $^{8}$

\section{LIMITATIONS}

There are several challenges in applying QI methodologies in surgery. The range of patient problems, procedures, and unexpected events make it difficult to apply QI initiatives that rely on minimizing variability. There can also be difficulty in conducting research into QI. The Hawthorne effect is a process where individuals modify their behavior due to their awareness of being observed. As applied to QI initiatives, the Hawthorne effect can result in increased productivity during the observation period that dissipates after the observation period is over, making it difficult to discern the true effects of the proposed intervention. ${ }^{3,5}$

Given the research conducted in the field thus far, there appears to be a role for QI in surgery. Although there are examples in the literature of successful QI initiatives in surgical practice, high quality studies are required to support evidence-based management for specific indications as current studies and interventions are heterogeneous and difficult to apply broadly.

\section{CONCLUSION}

Reducing system inefficiencies in the surgical setting is necessary in a publicly funded healthcare system with limited resources. Hospitals comprise the largest proportion of health care expenditures in Canada ${ }^{2}$ and OR activities tend to be the most resource intensive, thereby making it an attractive target for QI applications. 
Given the economic climate both nationally and internationally, QI methodologies in surgery constitute an emerging practice that should be considered at all levels of healthcare organizations, as they contribute to the financial viability and sustainability of the Canadian healthcare system for future generations.

\section{REFERENCES}

1. Nicolay CR, Purkayastha S, Greenhalgh A, et al. Systematic review of the application of quality improvement methodologies from the manufacturing industry to surgical healthcare. Br J Surg. 2012 Mar;99(3):324-35.

2. Canadian Institute for Health Information. National Health Expenditure Trends, 1975 to 2016. Ottawa, ON: CIHI; 2016.

3. Mason SE, Nicolay CR, Darzi A. The use of Lean and Six Sigma methodologies in surgery: a systematic review. Surgeon. 2015 Apr;13(2):91100.

4. Barbagallo S, Corradi L, Goyet JV, et al. Optimization and planning of operating room theatre activities: an original definition of pathways and process modeling. BMC Med Inform Decis Mak. 2015 May;15(38):1-16.

5. Collar RM, Shuman AG, Feiner S, et al. Lean management in academic surgery. J Am Coll Surg. 2012 Jun;214(6):928-36.

6. Stoutzenberger TL, Kitner SA, Ulrich BL, et al. Using Lean strategies to improve operating room efficiency. OR Manager. 2014 Jan;30(1):1820.

7. Valsangkar NP, Eppstein AC, Lawson RA, et al. Effect of Lean Processes on Surgical Wait Times and Efficiency in a Tertiary Care Veterans Affairs Medical Center. JAMA Surg. 2016 Sep 7. [Epub ahead of print]

8. Cima RR, Brown MJ, Hebl JR, et al. Use of lean and six sigma methodology to improve operating room efficiency in a high-volume tertiary-care academic medical center. J Am Coll Surg. 2011 Jul;213(1):83-92.

9. Fong AJ, Smith M, Langerman A. Efficiency improvement in the operating room. J Surg Res. 2016 Aug;204(2):371-83.

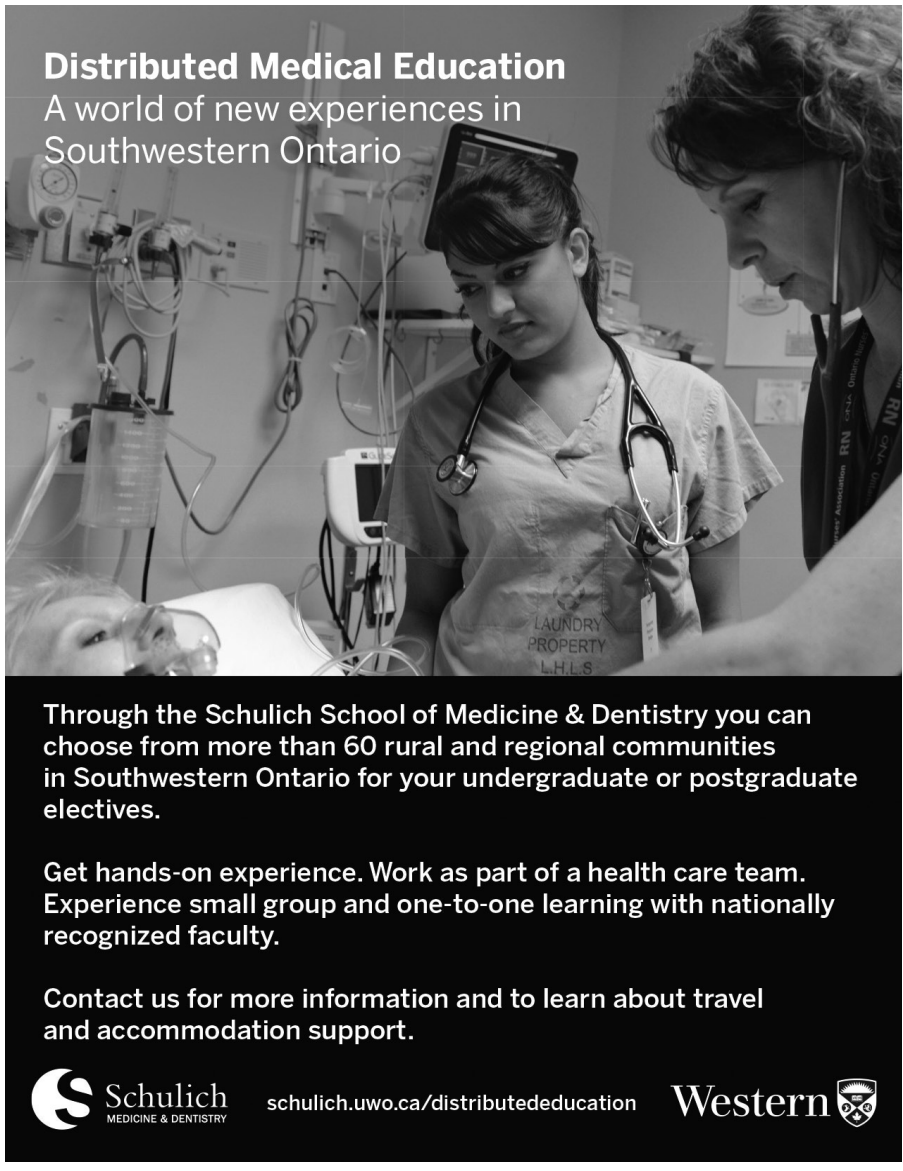

